\title{
Evaluación del comportamiento de sistemas de ventilación por captación lateral sobre baños de tratamiento de superficies: Condicionantes del proyecto de instalaciones ${ }^{(\cdot)}$
}

\author{
Enrique González*, Agustín Miñana*, Antonia Baeza*, Francisca Morales* y \\ Francisco Marzal**
}

\begin{abstract}
Resumen En la ventilación por extracción localizada aplicada a baños de tratamiento de superficies, el mejor elemento de control es aquel que permite el encerramiento total de la fuente emisora, siendo este procedimiento el punto de partida del proyectista para el diseño preliminar de la instalación. Ahora bien, la frecuente necesidad de introducir y extraer las piezas a tratar por su zona superior, mediante grúas móviles u otros dispositivos de carga, la reposición de los componentes del baño y la vigilancia por parte de los trabajadores, hace necesario que el acceso por dicha zona deba ser diáfano, imposibilitándose el encerramiento total. En consecuencia, deben eliminarse aquellos componentes que impidan llevar a cabo las citadas operaciones, recurriendo a la instalación de elementos de captación lateral. Otros inconvenientes del sistema, derivados del carácter omnidireccional de la aspiración, hacen necesario trabajar con elevados caudales para generar adecuadas velocidades de control en los dominios de los baños, por lo que no es aconsejable su implantación para longitudes superiores a 1,20 m. Por otro lado, la complejidad mecanística de los flujos de aire involucrados aconseja el empleo de dispositivos experimentales que permitan la visualización de las diferentes corrientes de aire implicadas, utilizando gases trazadores para evaluar cuantitativamente su eficacia. En este trabajo se plantean, en función de las investigaciones llevadas a cabo, las bases del diseño de instalaciones de ventilación a escala real que permita estudiar en profundidad la influencia de las variables que condicionan la eficacia de captación.
\end{abstract}

Palabras clave Ventilación de tanques abiertos. Elementos primarios de captación local. Visualización de flujos. Trazadores de gas. Eficacia de captura.

\section{Evaluation of the performance of exhaust local ventilation systems in treatment surface tanks: Constraint of the facilities project}

\begin{abstract}
In the design of local exhaust ventilation systems for open surface tanks, the element of control that permits the total shutting in the source is clearly the best, being this procedure the starting point for industrial projector for the preliminary design installation. However, the frequent need of introducing and extracting the workpieces by their upper side through mobile crane or other load devices, the reinstatement components of the tank and the surveillance on the part operators, makes necessary that the access in such zone side be easy, being disabled the total encirclement. Consequently those components that prevent carry out the cited operations should be eliminated, appealing to the installation of local exhaust ventilation. Other inconvenients of these systems, derivated from the character omnidirectional of the aspiration, make necessary to operate with high exhaust flows to generate suitable speeds of control in the baths, therefore it is not advisable their installation for lengths higher than $1.20 \mathrm{~m}$. On the other hand the complexity of the mechanism of the involved air flows advises the experimental devices employment that permit the visualisation of the different current of involved air, being furthermore necessary to use tracer gases to evaluate quantitatively the capture efficiency of the system. In this work are outlined, in function of the investigations carried out, the basis of the design of ventilation equipments to real scale that permit to study in-depth the influence of the variables that condition the capture efficiency.
\end{abstract}

Keywords Open surface tanks ventilation. Local exhaust hoods. Tracer gas. Flow visualisation. Capture efficiency.

(•) Trabajo recibido el día 12 de junio de 1999 y aceptado en su forma final el 29 de noviembre de 1999.

$\left(^{*}\right)$ Dpto. de Ingeniería Química. Universidad de Murcia. 30071 Espinardo. Murcia (España).

(**) Dpto. de Ingeniería Térmica y de Fluidos. Universidad Politécnica de Cartagena. 30203 Cartagena. Murcia (España). 


\section{INTRODUCCIÓN}

El diseño de una instalación experimental, representativa de los equipos y situaciones reales, requiere la consideración de los sistemas utilizados en la práctica habitual y, a la vez, de los factores que permitan garantizar la adecuación del dispositivo a la finalidad buscada.

En este caso, se trata de evaluar la eficacia de los sistemas de ventilación por captación lateral sobre baños de tratamiento de superficies mediante una instalación piloto. Para alcanzar este fin es necesario tener en cuenta determinados aspectos relativos; por un lado, la ventilación localizada y la captación lateral y, por otro, la visualización de flujos y la determinación de eficacias, pues ambos inciden en la configuración de la instalación y, por tanto, resultan ser factores condicionantes del diseño de la misma.

\section{VENTILACIÓN LOCALIZADA}

Se basa en encerrar o capturar el contaminante o flujo emisivo muy cerca de la fuente donde se genera. Comparadas con la ventilación por dilución, las extracciones localizadas necesitan conducir caudales de aire mucho más pequeños y, en general, la eficacia del sistema es más elevada, al impedir la dispersión de la emisión. Desde la perspectiva económica, el coste de los sistemas de extracción es más elevado que el de la ventilación general, pero, en muchas ocasiones, los costes operacionales pueden compensar el balance si resultara necesario el acondicionamiento térmico del aire procedente del exterior.

El sistema consta de tres elementos básicos interconectados ${ }^{[1-3]}$ : el elemento primario de captación, con la geometría y disposición adecuadas para el encerramiento, recepción o captación del flujo emisivo; un equipo de depuración que retenga o transforme los contaminantes, con la eficacia suficiente para satisfacer las exigencias emisivas al medio ambiente exterior; y un ventilador que proporcione los caudales para llevar a cabo con éxito la operación.

Los diferentes elementos primarios de captación se integran en cuatro grupos: encerramientos y cabinas, receptores, captadores y sistemas combinados de impulsión-aspiración. En los primeros, el foco de emisión está situado en el interior de sus límites geométricos, y el caudal de aspiración debe provocar velocidades de aire en la áreas de entrada que superen la velocidad de escape de los contami- nantes ${ }^{[4]}$. Así pues, la velocidad del aire en la entrada actúa como una cortina dinámica que impide la salida del aire interior. En los restantes grupos, los focos están situados fuera de los límites de los elementos primarios, diferenciándose en la situación de las áreas abiertas respecto al flujo emisivo y por sus comportamientos operativos. Así, en los receptores, la entrada de aspiración se sitúa perpendicularmente a la dirección de la emisión, siendo característicos los utilizados en procesos térmicos o mecánicos ${ }^{[5-7]}$. En ellos se trabaja con un caudal algo superior al procedente del foco emisor, al alcanzar la entrada del elemento, sin necesidad de generar en el exterior de los mismos campos de velocidad significativos. Por el contrario, los captadores, entendidos como los que se encuentran situados adyacentes al foco de contaminante ${ }^{[7-9]}$, deben generar corrientes exteriores de aire capaces de arrastrar el flujo contaminante hacia ellos, lo que implica la aspiración de caudales más elevados que los anteríiores, son los de menor eficacia, al estar afectados, en mayor o menor medida, por las corrientes aleatorias exteriores. En los sistemas combinados de impulsión-aspiración, la impulsión de aire en forma de cortina circula paralelamente a la superficie del baño, arrastrando las emisiones hacia la aspiración.

\section{CAPTACIÓN LATERAL EN BAÑOS}

La información bibliográfica más relevante de estos sistemas de captación lateral (Fig. 1) está contenida en normas ${ }^{[10-13]}$ o en manuales ${ }^{[14]}$ que recomiendan los diseños geométricos e intervalos de las variables de trabajo más importantes.

La distribución de velocidades en zonas próximas a elementos de aspiración con entrada cuadrada difiere poco de la correspondiente a una circular. En ambas, la velocidad en el eje, $v$, puede
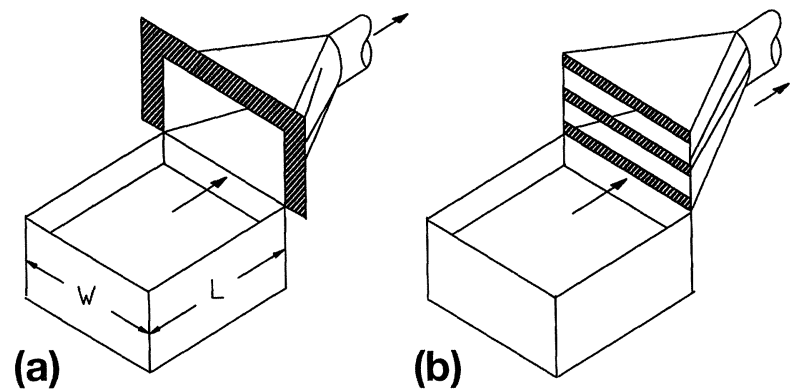

Figura 1. Formas de elementos primarios de captación. a) Entrada simple con pantalla. b) Entrada tipo rendija.

Figure 1. Types of lateral exhaust hoods. a) Simple hood with baffle. b) Slot hood. 
expresarse con aproximación aceptable mediante la expresión de Dalla Valle ${ }^{[14]}$ :

$$
v=\frac{Q_{a}}{10 x^{2}+A}
$$

donde:

$\mathrm{Q}_{a}=$ Caudal aspirado.

$x=$ Distancia medida en el eje perpendicular a la entrada.

$\mathrm{A}=$ Área de entrada.

La ecuación (1), válida para valores de $x$ inferiores a 1,5 veces el diámetro de la entrada circular o el lado, si se trata de una superficie cuadrada, proporciona una información muy limitada, dado que para su aplicación a la captación de focos extensos -por ejemplo, baños- se precisa conocer la distribución de velocidades en todo el dominio de la emisión. Una forma de abordar este problema consiste en dividir el área de entrada en una serie de celdillas cuadradas y evaluar en cada punto exterior la velocidad inducida por cada una de ellas, realizando la suma vectorial correspondiente para determinar el módulo y dirección de la velocidad final. Es evidente la necesidad de abordar el problema mediante cálculo computacional; así, se obtendrían los campos de velocidades representados mediante superficies isocinéticas y líneas de flujo, como se representa en la figura 2 , donde se incluyen genéricamente sólo las correspondientes a uno de los planos de simetría.

Si la entrada del elemento de captación es rectangular, como es habitual en los baños, las superficies isocinéticas son elipsoides cuyos ejes están asociados a los lados mayor y menor del rectángulo (Fig. 3).

Como se observa, los dominios definidos por las diferentes líneas isocinéticas son pequeños, por lo que para ampliarlos se puede actuar en tres direcciones:

- Incrementando el caudal. Esta opción está condicionada por factores económicos y operacionales, ya que condiciona las dimensiones de las conducciones, ventilador y depurador, incrementando los niveles de ruido y el coste del acondicionamiento térmico.

- Implantando tabiques deflectores, paralelos a la superficie de la entrada (Fig. 1a), perpendiculares -esto es, por extensión de las paredes laterales-, o mixtos (Fig. 4). De esta forma se limita la captación de aire procedente de las partes posterior y laterales del elemento, pero, en todo

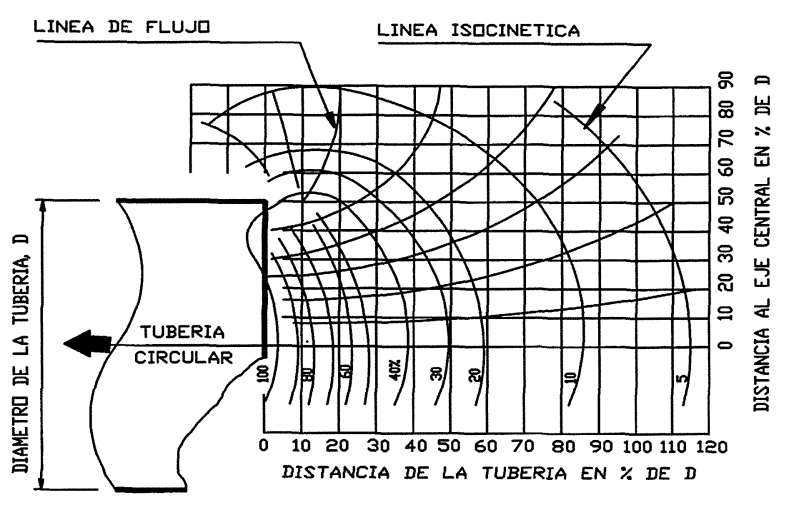

(a)

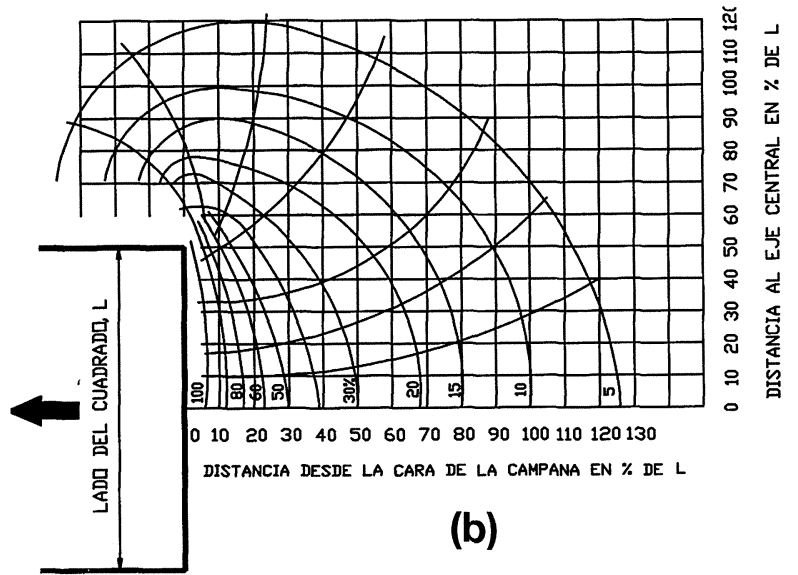

Figura 2. Líneas de flujo e isocinéticas correspondientes a un plano de simetría de una entrada circular (a) y cuadrada (b). El porcentaje de las líneas isocinéticas se refiere al valor de la velocidad respecto a la de entrada en la conducción.

Figure 2. Velocity contours and flow directional-lines in front of circular suction pipe (a) and square suction hood (b). The percentage of the isocinetic lines is referred to value of the speed with respect to that of entry in the conduct.

caso, la influencia de estas actuaciones no consigue incrementos de velocidad suficientes para resolver los problemas reales, como queda representado en la figura 5 , donde se comparan las mismas líneas isocinéticas para dos entradas, con pantalla y sin ella.

- La utilización de entradas tipo ranura (Fig. 1b) es una alternativa interesante por dos razones: en primer lugar, porque al disminuir el área de entrada aumenta la velocidad en las rendijas y este incremento se transmite a la zona exterior de influencia, como puede deducirse a través de los comportamientos descritos y, por otro lado, se consigue mayor uniformidad de la velocidad a lo largo de la rendija. Como contrapartida, se incrementa la pérdida de carga respecto a la entrada simple.

Otro aspecto a tener en cuenta en la captación lateral en baños es la influencia favorable de la 


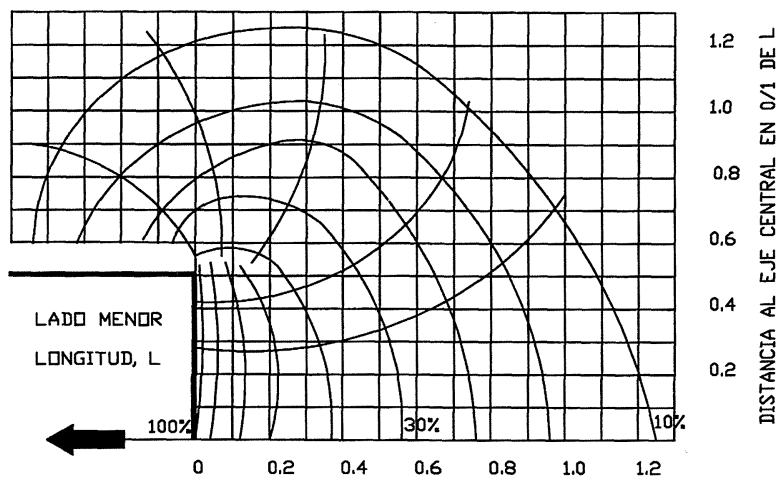

DISTANCIA DESDE LA CARA DE LA CAMPANA EN $\mathrm{D} / 1$ DE L

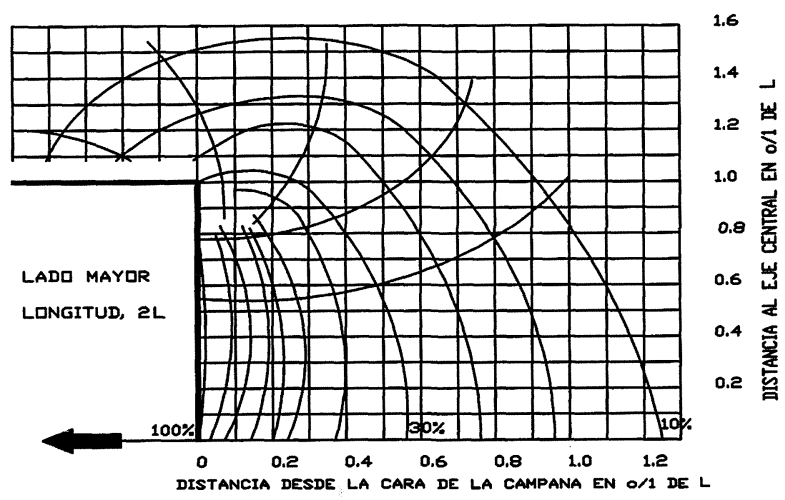

Figura 3. Líneas de flujo e isocinéticas en una entrada rectangular de relación 1:2. El porcentaje de las líneas isocinéticas se refiere al valor de la velocidad respecto a la de entrada en la conducción.

Figure 3. Velocity contours and flow directional-lines in principal centerline planes of rectangular suction hood with side ratio 1:2. The percentage of the isocinetic lines is referred to value of the speed with respect to that of entry in the conduct.

superficie de estos, que actúa como pantalla horizontal, modificando el campo de velocidades de forma similar a como lo haría una entrada de anchura

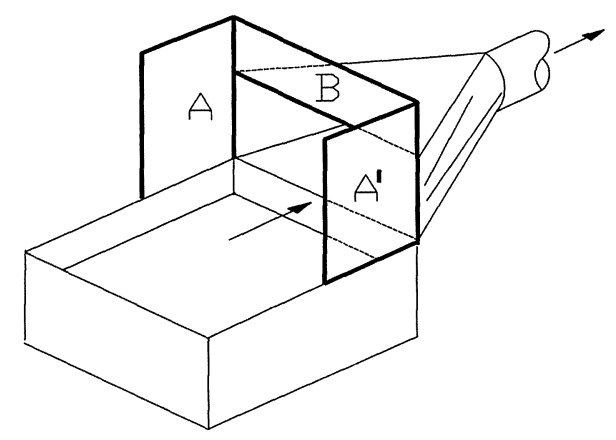

Figura 4. Captación lateral en baño dotada de pantallas laterales, A y $A^{\prime}$ y frontal, B.

Figure 4. Exhaust hood with lateral baffles, $A$ and $A^{\prime}$, and frontal baffle, $B$.

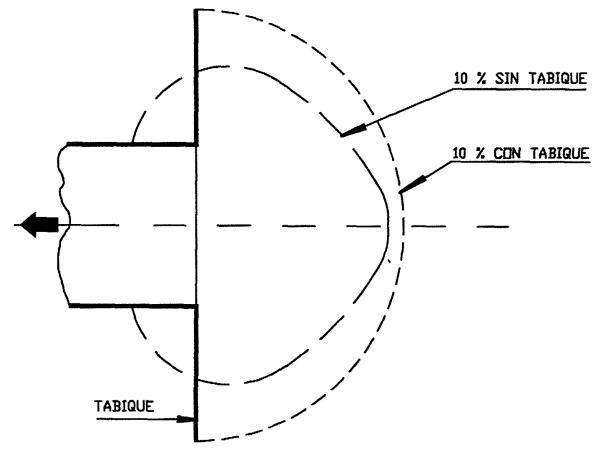

Figura 5. Líneas isocinéticas en entradas de aspiración simple y con pantalla. (El porcentaje se refiere al valor de la velocidad respecto a la producida en la entrada de la conducción).

Figure 5. Effect of hood flanks on velocity contours.

doble, tal y como se indica en la figura 6 , lo que supone un mayor incremento de la velocidad en las proximidades de la superficie.

El diseño geométrico del pleno juega un papel fundamental para lograr la uniformidad de la velocidad de la entrada, aconsejándose diseñar la conexión troncocónica con un ángulo no inferior a $60^{\circ}$ y máximo de $90^{\circ}$ (Fig. 7a), dependiendo del factor de forma o relación entre su altura y anchura. De no ser así, la velocidad en la entrada quedaría distorsionada y, además, podrían formarse corrientes preferenciales de retroceso, con elevada probabilidad de ser expedidas al exterior. En este mismo sentido, para grandes baños se aconseja compartimentar las entradas (Fig. 7b).

Mientras que la American Conference of Gobernmental Industrial Hygienists ${ }^{[15]}$ propone la velocidad mínima de control para cada configuración de entrada, el Institut National de Recherche et de Sècurité ${ }^{[13]}$ ha establecido unas recomendaciones para la selección de los caudales de aspiración por metro cuadrado de superficie de baño

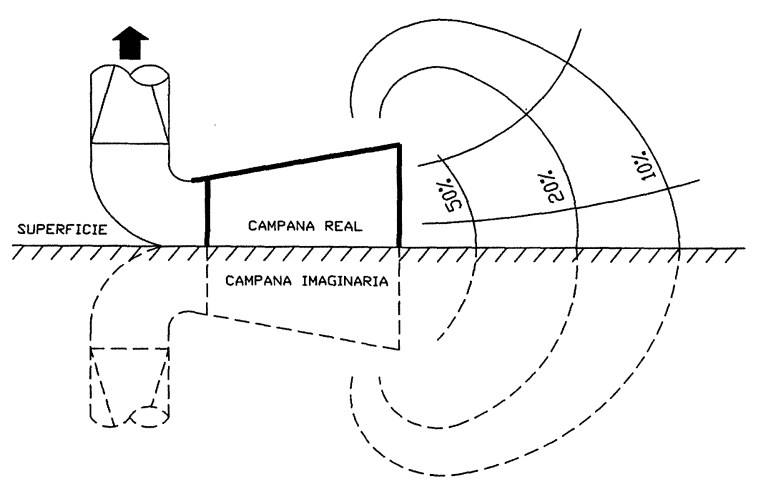

Figura 6. Líneas isocinéticas generadas por una entrada de aspiración simple apoyada sobre una superficie horizontal.

Figure 6. Velocity contours of hood resting on table. 

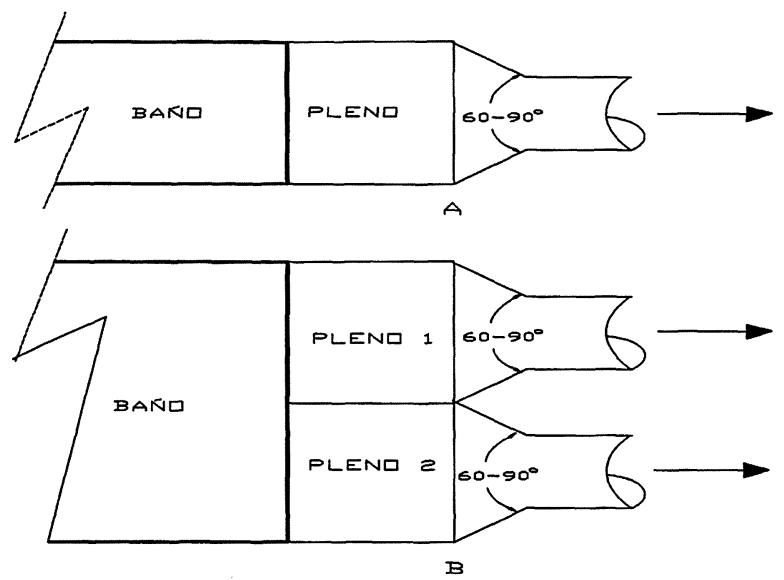

Figura 7. Vista en planta de captaciones laterales simples para conseguir velocidades uniformes en las entradas.

Figure 7. Plans view of simple lateral exhaust to obtain uniform speeds in air intake.

dependiendo de las clases de riesgo y de la relación anchura/longitud del baño, para distintos dispositivos de captación lateral.

\section{VISUALIZACIÓN DE FLUJOS Y DETERMINA- CIÓN DE EFICACIAS}

Para conocer la incidencia de las variables que intervienen en una instalación concreta de ventilación es necesario recurrir a métodos cualitativos -mediante técnicas de visualización-, y cuantitativos -medidas de velocidades, caudales, concentraciones, etc.- que permitirán el mejor conocimiento de los flujos involucrados y el ajuste del sistema para obtener las disposiciones más eficaces.

Algunas técnicas de visualización de flujos emplean el vapor de agua generado en un baño ${ }^{[16-17]}$, niebla ${ }^{[18-19]}$, como puede verse en la figura 8 , técnicas fotográficas ${ }^{[20]}$, etc, empleando gases trazadores habitualmente como métodos cuantitativos ${ }^{[21-23]}$.

Los gases trazadores más utilizados son el óxido de dinitrógeno, dióxido de carbono, hexafluoruro de azufre, freones y helio ${ }^{[7 \text { y } 24-25]}$. El hexafluoruro de azufre es uno de los más empleados por su bajo nivel de toxicidad, especificidad y posibilidades de medida a niveles de partes por millón mediante infrarrojos ${ }^{[18 \text { y 26-27] }}$ e incluso a menores concentraciones con técnicas más sensibles ${ }^{[28-29]}$, lo que permite disminuir el flujo de trazador en grandes instalaciones de ventilación.

La eficacia de la captación se define como la relación de flujos de contaminante captado por el sistema y el generado por el foco emisor ${ }^{[8 \text { y } 30 \mid}$. Para su determinación se requiere realizar dos de las si-

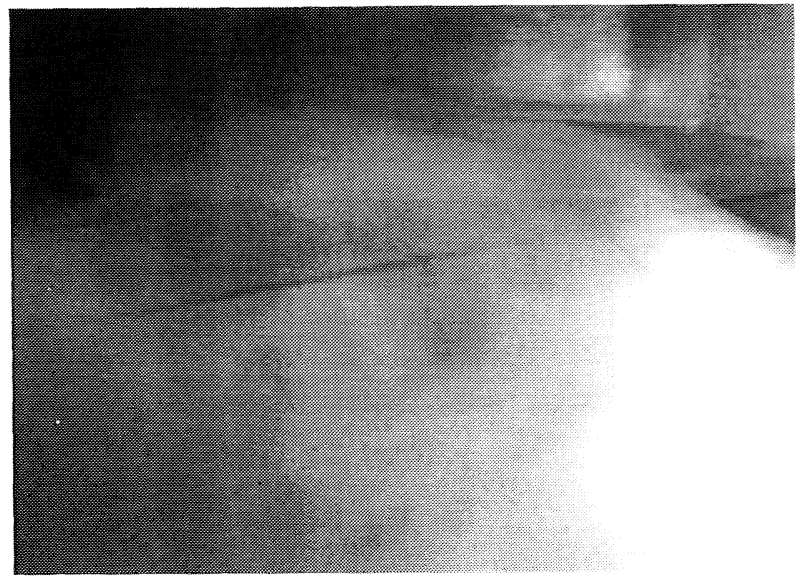

Figura 8. Incorporación de la corriente de aspiración al elemento primario de captación (niebla inyectada desde el baño).

Figure 8. Incorporation of the exhaust flow to the primary captation element (mist injected from the bath).

guientes medidas: el flujo generado, $\mathrm{G}_{\mathrm{T}}$; el captado, $\mathrm{G}_{\mathrm{a}} \dot{y}$, el emitido al ambiente exterior, $\mathrm{G}_{\mathrm{p}}$ (esto es, el no captado), ya que, en régimen estacionario:

$$
\mathrm{G}_{\mathrm{T}}=\mathrm{G}_{\mathrm{a}}+\mathrm{G}_{\mathrm{p}}
$$

La medida de los flujos de contaminantes generados en baños reales presenta numerosas dificultades debidas a la heterogeneidad de los componentes, cuya composición puede variar con el tiempo, y a la dinámica temporal de la propia emisión, típica de procesos no estacionarios. Aún más compleja resulta la medida del flujo emitido al recinto donde se ubica el baño, dada la dispersión y dilución provocada por las corrientes de aire y los movimientos convectivos internos, además de la imposibilidad de segregar las emisiones de otros procesos anexos. Todo ello justifica las siguientes recomendaciones:

- Utilizar trazadores diferentes a los emitidos por el proceso, que sean insolubles y no reaccionen con los componentes del baño. Esta condición limita la utilización de los sistemas reales si se requieren determinaciones cuantitativas precisas, dado que el concepto de insolubilidad no es absoluto cuando las concentraciones puestas en juego son muy pequeñas. Basta una pequeña absorción del trazador por parte de los componentes del baño para afectar al balance másico de éste en el sistema de ventilación. Así pues, es aconsejable la utilización de instalaciones experimentales que no presenten estos problemas. 
- Conviene que la emisión superficial sobre el baño sea uniforme para evaluar correctamente la eficacia, puesto que ésta varía a lo largo de la longitud del baño, siendo tanto menor cuanto mayor sea la distancia entre los focos emisores puntuales al elemento primario de aspiración.

- El objetivo final del sistema de ventilación es evitar que las concentraciones de los contaminantes en el recinto superen los valores legales o recomendados. En este sentido, podría pensarse en medir los niveles de inmisión como parámetro para determinar la eficacia de captación, pero esta metodología presenta dos inconvenientes: en primer lugar, la concentración exterior evoluciona espacial y temporalmente, planteando discontinuidades y, por otro lado, el nivel de inmisión depende de las características geométricas del recinto y de la fluidodinámica del aire.

Por todo ello, es más conveniente efectuar las medidas en el sistema de captación de la instalación piloto, tal y como se representa en la figura 9.

En la figura 9a el flujo de trazador se introduce directamente en la conducción de aspiración, con el sistema funcionando bajo unas determinadas condiciones de trabajo, efectuándose la medida de la concentración, $C_{a}$, en el equipo indicado, situado en una zona alejada donde se garantice la total homogeneización de la mezcla trazador-aire. Dicha concentración representa el valor de referencia -máximo-.
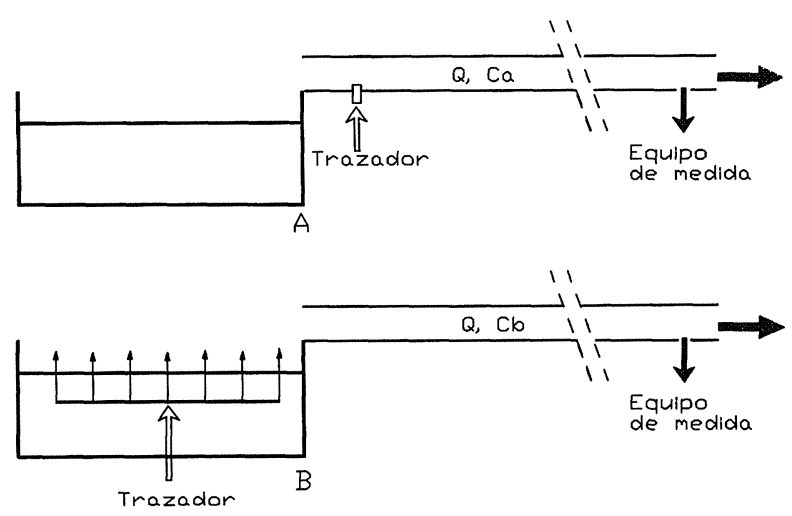

Figura 9. Disposición para determinar la eficacia total de captación. a) El trazador se inyecta en la tubería de aspiración. b) El trazador se inyecta uniformemente desde la superficie del baño.

Figure 9. Method used to measure capture efficiency using tracer gas technique. a) Value corresponding to total tracer gas emission in duct. b) Value corresponding to tracer gas emission simulating pollutant emission.
En la figura 9b, el mismo flujo de trazador se introduce distribuido uniformemente desde la superficie del baño, sin variar las condiciones operativas, determinándose la concentración, $C_{b}$, en el mismo lugar.

La eficacia del sistema, $E_{t}$, viene dada por la expresión:

$$
E_{t}=\frac{C_{b}}{C_{a}}
$$

Es evidente el interés que presenta el diseño de los elementos del sistema que permitan una adecuada aplicación de los métodos cualitativos y cuantitativos de visualización y de determinación de eficacias aquí descritos.

\section{REFERENCIAS}

[1] M. Esparza, J.E. FloRes, O.P. SÁnchez y R.E. Pocovi, Ing. Quím. 5 (1990) 207-211.

[2] P.L. MendaZA, NT-265. Instituto Nacional de Seguridad e Higiene en el Trabajo, Barcelona, España, 1991.

[3] R.H. PerRY, Manual del Ingeniero Químico. 6a ed. McGraw-Hill, México 1992.

[4] B. Fletcher y A.E. Johnson, Ann. Occup. Hyg. 25 (1982) 365-372.

[5] J.L. ALDEN y J.M. KANE. Design of Industrial Ventilation Systems. 5th ed. Industrial Press Inc. New York, EE.UU, 1982 pp. 281.

[6] R. BERGLUND, Proc. Int. Conf. Interfinish 92. 1992 pp. $1538-1152$.

[7] R. BRACONNIER, Cahier de notes documentaires, CDU 621.794, ND 1841-144-91, 1991 pp. 463-478.

[8] B. FletCHer y A.E. JOHnson, Proc. Int. Symp. on Ventilation for Contaminant Control. ACGIH. Canadá, 1985 pp. 333-353.

[9] H.E. MCLOONE, S.E. GufFey y J.P. CuRRAn, Am. Ind. Hyg. Assoc. J. 54 (1993) 87-94.

[10] OSHA, Occupational Safety and Health Administration. General Industry Standars. 1910.94 (d) (3) \& (4), 1985.

[11] ANSI, American National Standards Institute. Fundamentals Governing. Standards: Z9.2-1979 (R1991).

[12] ASHRAE, American Society of Heating, Refrigerating and Air Conditioning Engineers. Handbook of Fundamentals. New York, EE.UU., 1985.

[13] INRS, Institut National de Recherche et de Sécurité. Guide Practique de Ventilation, 2. ED 651. Paris, Francia, 1989.

[14] J. Dalla Valle, Exhaust Hoods, Industrial Press, New York, EE.UU., 1952

[15] ACGIH, American Conference of Governmental Industrial Hygienist. Ventilación Industrial. I ed. Generalitat Valenciana. Valencia, España, 1992.

[16] W.P. BATISTA, Heat. Pip. Air Cond. 1 (1947) 85-89.

[17] A.G. SAG, ASHRAE Transactions: Symposia. CH-93-91. 1993 pp. 971-978.

[18] J.N. WoOds y J.S. MCKarns, Am. Ind. Hyg. Assoc. J. 56 (1995) 1208-1214. 
[19] E. González, A. Miñana, F. Morales, M. Rodríguez y F. MARZAL, Montajes Instalaciones (324) (1999) 89-94.

[20] R.J. Heinsohn, S.T. YU, C.L. MerkLe, G.S. Settles y C. HuItema, Proc. Int. Symp. on Ventilation for Contaminant Control. ACGIH. Canadá. 1985 pp. 529-566.

[21] L.J. Flanigan, S.G. Talbert, D.E. Semones y B.C. Kim, National Institute for Occupational Safety. Contract $n^{\circ}$ HSM 099-71-61, Cincinnati. Ohio, OH, EE.UU. 1974, pp. 1-66.

[22] D.J. Huebener y R. Hughes, Am. Ind. Hyg. Assoc. J. 46 (1985) 262-267.

[23] M.K. KLeIN, Am. Ind. Hyg. Assoc. J. 47 (1986) 369373.

[24] R. Niemelä, A. Lefevre, J.P. Muller y G. Aubertin, Ann. Occup. Hyg. 35 (1991) 405-417.
[25] D. Bemer, J.P. Muller y J.M. Dessagne, Indoor Air 8 (1998) 47-60.

[26] J.A. Mosovsky, Am. Ind. Hyg. Assoc. J. 56 (1995) 4449.

[27] E. González, A. Miñana, F. Morales, M. Rodríguez y F. MARZAL, Ing. Quím., (362) (1999) en prensa.

[28] C. IRWIN, R. EdWARDS y P.J. Burberry, Proc. Int. Symp. on Ventilation for Contaminant Control. ACGIH. Canadá. 1985, pp. 245-250.

[29] L.M. ConRoy y M. EllenbeCKer, J. Appl. Ind. Hyg. 4 (1989) 135-142.

[30] M.J. EllenbeCKer, R.F. GeMPel y W.A. Burguess, Am. Ind. Hyg. Assoc, J. 44 (1983) 752-755. 